ARTICLE

\title{
Low threshold lasing emissions from a single upconversion nanocrystal
}

\author{
Yunfei Shang (10) ${ }^{1,2,3}$, Jiajia Zhou (10 ${ }^{1 凶}$, Yangjian Cai ${ }^{3}$, Fan Wang (10 ${ }^{1}$, Angel Fernandez-Bravo ${ }^{4}$, Chunhui Yang ${ }^{2}$,
} Lei Jiang (10) ${ }^{5} \&$ Dayong Jin (10 ${ }^{1,3}$

Cross-relaxation among neighboring emitters normally causes self-quenching and limits the brightness of luminescence. However, in nanomaterials, cross-relaxation could be wellcontrolled and employed for increasing the luminescence efficiency at specific wavelengths. Here we report that cross-relaxation can modulate both the brightness of single upconversion nanoparticles and the threshold to reach population inversion, and both are critical factors in producing the ultra-low threshold lasing emissions in a micro cavity laser. By homogenously coating a $5-\mu \mathrm{m}$ cavity with a single layer of nanoparticles, we demonstrate that doping $\mathrm{Tm}^{3+}$ ions at $2 \%$ can facilitate the electron accumulation at the intermediate state of ${ }^{3} \mathrm{H}_{4}$ level and efficiently decrease the lasing threshold by more than one order of magnitude. As a result, we demonstrate up-converted lasing emissions with an ultralow threshold of continuous-wave excitation of $\sim 150 \mathrm{~W} / \mathrm{cm}^{2}$ achieved at room temperature. A single nanoparticle can lase with a full width at half-maximum as narrow as $\sim 0.45 \mathrm{~nm}$.

\footnotetext{
${ }^{1}$ Institute for Biomedical Materials and Devices (IBMD), Faculty of Science, University of Technology Sydney, Sydney, NSW 2007, Australia. ${ }^{2}$ MIIT Key Laboratory of Critical Materials Technology for New Energy Conversion and Storage, School of Chemistry and Chemical Engineering, Harbin Institute of Technology, Harbin 150001, P.R. China. ${ }^{3}$ UTS-SUStech Joint Research Centre for Biomedical Materials and Devices, Department of Biomedical Engineering, Southern University of Science and Technology, Shenzhen, Guangdong, P.R. China. ${ }^{4}$ Department of Physics, Politecnico di Milano, Piazza L. Da Vinci 32, Milano 20133, Italy. ${ }^{5}$ Laboratory of Bio inspired Smart Interface Science, Technical Institute of Physics and Chemistry, Chinese Academy of Sciences, Beijing 100190, P.R. China. ${ }^{凶}$ email: jiajia.zhou@uts.edu.au
} 
C ross-relaxation $(\mathrm{CR})$ is an energy transfer process between a pair of nearby emitters, where one at a higher excited energy state transfers its photon energy to the other one at a lower excited state or ground state, so that both can simultaneously reach their intermediate excited states ${ }^{1}$. This process can be often observed in lanthanides ions those featured with a series of sophisticated intermediate energy levels and each with long lifetimes ${ }^{2,3}$. CR accumulates energy in the intermediate excited states, as a conducive process to the formation of population inversion that is essential for the generation of lasing emissions. This explains why lanthanide ions doped laser crystals and glass are ascendant gain medium ${ }^{4-6}$. Though the concentration of lanthanide ions determines the extent of $\mathrm{CR}$ and thereby the threshold for lasing emission generation, in the bulk materials the doping uniformity and dynamic range of lanthanide ions are hard to control, e.g., typically $0.25-1 \%$ for $\mathrm{Tm}^{3+}$ in $\mathrm{YVO}_{4}, \mathrm{MgWO}_{4}$ or YAG laser crystal $\left(<8 \times 10^{20} \text { ions } / \mathrm{cm}^{3}\right)^{7-9}$. High doping in bulk crystal and glass often leads to the non-uniform distribution of the ions and localized excessive CR, which results in luminescence quenching, self-heating, and high laser threshold.

At the nanoscale, lanthanides doped upconversion nanoparticles (UCNPs) and wet chemistry synthesis strategies have been well developed, so that their size, shape and doping concentrations can be precisely controlled with high accuracies in both morphological and optical uniformities ${ }^{10-15}$. In a typical $\mathrm{NaYF}_{4}$ host, the doping percentage of a lanthanide ion can be arbitrarily tuned between zero and its unity to form the "alloyed" nanocrystals ${ }^{16,17}$. This allows several recent studies on the role of doping concentrations and the degree of CR between ions in increasing the efficiency of optical depletion in super-resolution microscopy and facilitating the generation of near-infrared and single band emissions ${ }^{2,18-22}$. Encouragingly, singly $\mathrm{Tm}^{3+}$ doped UCNPs with energy looping effect have been recently reported as an efficient gain medium to generate room-temperature continuous-wave (CW) pumped laser with a threshold of $\sim 14 \mathrm{~kW} / \mathrm{cm}^{2} 23$.

Here we study the role of CR (doping concentration), excitation power density and the size of a single nanoparticle, and to identify the optimum conditions and key characteristics to set a single nanoparticle to lase. We find that the brightness of UCNPs and its efficiency in establishing the population inversion as the gain medium as well as the quality factors of a typical whisperinggallery-mode (WGM) cavity can be strongly affected by the doping concentration, excitation power density and the size of UCNPs when coating a typical polystyrene microsphere by a single layer of self-assembled UCNPs or only a single UCNP. In the classical $\mathrm{Yb}^{3+}-\mathrm{Tm}^{3+}$ co-doped energy transfer system, we find that the higher doping concentration of $\mathrm{Tm}^{3+}$ facilities higher probability for $\mathrm{CR}$, but requires higher laser excitation intensity to produce sufficiently bright upconversion emissions. Moreover, though the larger size of UCNPs typically produces stronger emissions, it generates a stronger scattering loss by the increased roughness of the cavity surface. Since the scattering scales with the 6th power of the particles' diameter, reducing the number of particles will significantly reduce the loses of the cavities $^{24,25}$. This effect can significantly reduce the efficiency for micro cavity lasers coated by a single layer of self-assembled UCNPs, but has negligible influence on the lasing emissions from a single UCNP.

\section{Results and discussion}

Population inversion property of CR nanoparticles. TEM images displayed the Fig. 1a of a series of morphology-uniform UCNPs at different $\mathrm{Tm}^{3+}$ concentrations show the monodispersity of our nanocrystals. Within the volume of $\sim 7240 \mathrm{~nm}^{3}$, in a $24 \mathrm{~nm}$ UCNP (Supplementary Fig. 1), we tune the amount of the $\mathrm{Tm}^{3+}$ ions from $\sim 200$ to $\sim 8000,0.2 \mathrm{~mol} . \%$ to $8 \mathrm{~mol} . \%$ correspondingly. As shown in Fig. 1b, when the concentration of $\mathrm{Tm}^{3+}$ is fixed at low level, the population inversion purely depends on the high power density of the pumping laser, as the activation of the emitters is mainly through the sensitization of $\mathrm{Yb}^{3+}$ (Supplementary Fig. 2) and there is negligible CR due to the long distance between the lanthanide ions, e.g., the averaged large distance can be calculated as $\sim 3.3 \mathrm{~nm}$ at the doping concentration of $0.2 \mathrm{~mol} . \%$. The dependence on the high power excitation can be alleviated by CRs, $\left({ }^{1} \mathrm{G}_{4},{ }^{3} \mathrm{H}_{6} \rightarrow{ }^{3} \mathrm{H}_{5},{ }^{3} \mathrm{H}_{4}\right),\left({ }^{1} \mathrm{G}_{4},{ }^{3} \mathrm{H}_{6} \rightarrow{ }^{3} \mathrm{~F}_{2,3}\right.$, $\left.{ }^{3} \mathrm{~F}_{4}\right),\left({ }^{3} \mathrm{~F}_{2,3},{ }^{3} \mathrm{~F}_{4} \rightarrow{ }^{3} \mathrm{H}_{4},{ }^{3} \mathrm{H}_{5}\right){ }^{18,26}$, which facilitates the establishment of population inversion at intermediate levels, such as ${ }^{3} \mathrm{H}_{4}$. Besides, highly $\mathrm{Tm}^{3+}$ doped UCNPs leads to the decrease in the $\mathrm{Yb}^{3+}-\mathrm{Tm}^{3+}$ distance and increase in the $\mathrm{Yb}^{3+}-\mathrm{Tm}^{3+}$ energy transfer efficiency. But this strategy, as illustrated in Fig. 1d, could lead to a quenching of the overall upconversion emissions, when doping too many emitters. As shown in Supplementary Fig. 4, the decrease in the $\mathrm{Yb}^{3+}$ lifetime values and the trend of energy transfer efficiency saturation appeared at the very large $\mathrm{Tm}^{3+}$ concentrations (e.g., $>8 \mathrm{~mol} . \%$ ), indicate the possible $\mathrm{Tm}^{3+}-\mathrm{Yb}^{3+}$ energy back transfer ${ }^{27}$. To alleviate the concentration quenching and energy back transfer effects in the highly doped UCNPs, high excitation power density is required to pump the significant amount of the ground level $\mathrm{Tm}^{3+}$ emitters and $\mathrm{Yb}^{3+}$ sensitizers. These suggest the existence of a sweet spot of optimum $\mathrm{Tm}^{3+}$ doping concentration and excitation pumping power for the low threshold establishment of population inversion.

We first characterize the upconversion emission spectra of the series of $24 \mathrm{~nm}$ UCNPs (Fig. 1a). Diversified spectral distributions are observed when varying the $\mathrm{Tm}^{3+}$ concentrations (Fig. 2a), suggesting the concentration-dependent $\mathrm{CR}$ effect. The proportions of the emissions from ${ }^{3} \mathrm{H}_{4}$ to ${ }^{3} \mathrm{H}_{6}$ transition show an upward tendency with the increase of $\mathrm{Tm}^{3+}$ concentration, which indicates the CR induced efficient energy accumulation (Supplementary Fig. 6). At the low concentration range, the energy, sensitized by and transferred from $\mathrm{Yb}^{3+}$, is distributed onto all the excited states, including ${ }^{1} \mathrm{D}_{2},{ }^{1} \mathrm{G}_{4}$, and ${ }^{3} \mathrm{H}_{4}$ (Supplementary Fig. 7). When the $\mathrm{Tm}^{3+}$ concentration increases from $1 \mathrm{~mol} . \%$ to 2 mol.\%, the peak intensities associated with the ${ }^{1} G_{4}$ level decrease, while the ${ }^{3} \mathrm{H}_{4}$ initiated transition intensity increases. Figure $2 \mathrm{~b}$ displays the quantitative intensity evolution and ratios as the function of $\mathrm{Tm}^{3+}$ concentration. The intensity at $802 \mathrm{~nm}$ $\left({ }^{3} \mathrm{H}_{4} \rightarrow{ }^{3} \mathrm{H}_{6}\right)$ transition, the intensity ratios of $802 \mathrm{~nm} / 473 \mathrm{~nm}$ and $802 \mathrm{~nm} / 645 \mathrm{~nm}$ reach their peak values at $2 \mathrm{~mol} \% \mathrm{Tm}^{3+}$ due to the $\mathrm{CR}$ induced population enrichment at the excitation power density of $100 \mathrm{~W} / \mathrm{cm}^{2}$. Further increase in the $\mathrm{Tm}^{3+}$ concentration, e.g. $4 \mathrm{~mol} \%$ and $8 \mathrm{~mol} \%$, depopulates the ${ }^{3} \mathrm{H}_{4}$ level with decreased intensity at $802 \mathrm{~nm}$ due to the excessive CR induced energy loss and the possible back energy transfer from $\mathrm{Tm}^{3+}$ to $\mathrm{Yb}^{3+}$. As $\mathrm{CR}$ involves different energy levels between a pair of nearby emitters, the population distribution of each energy level is strongly dependent on the excitation power density, so is the $\mathrm{CR}$ dynamics 2,28 . And Fig. 2c shows the power-dependent population of ${ }^{3} \mathrm{H}_{4}$ level by analyzing the peak intensity at 802 $\mathrm{nm}$. The $2 \mathrm{~mol} . \% \mathrm{Tm}^{3+}$ doped sample shows the highest intensities of $802 \mathrm{~nm}$ emissions within the excitation power density range of $0.6-150 \mathrm{~W} / \mathrm{cm}^{2}$ due to the CR effect. Above the power density of $10 \mathrm{~W} / \mathrm{cm}^{2}$, the low doping samples are saturated while the highly doped samples exhibit an accelerated growth trend of the emission intensities, indicating the non-linear transition dynamics caused by the power-dependent and concentration-dependent CR effect.

Characterization of microlasers coated with UCNPs. Figure $2 \mathrm{~d}$, e and Supplementary Fig. 8 present the typical images from SEM 
a

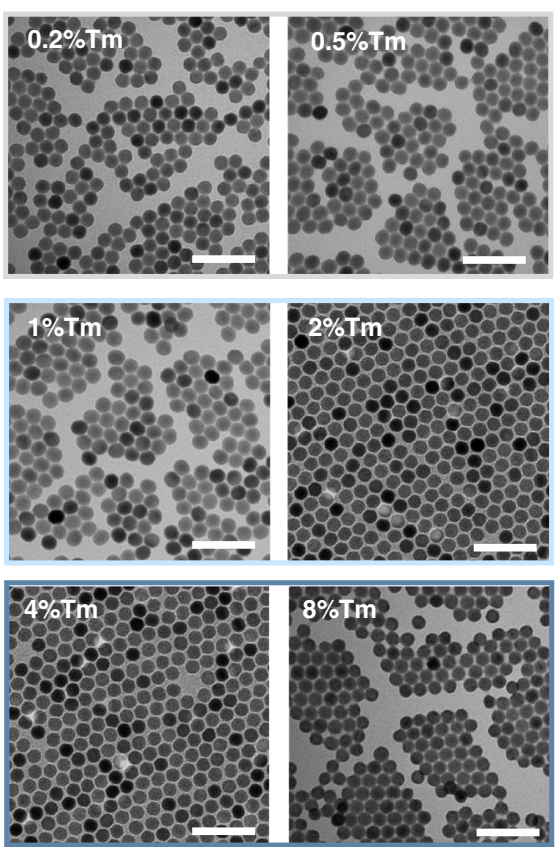

b

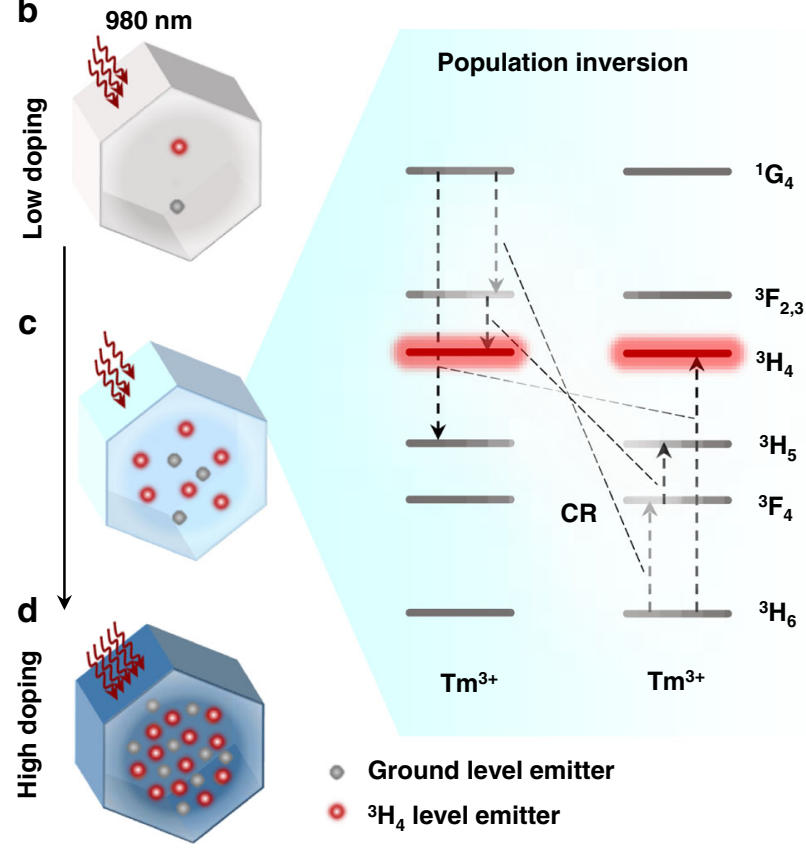

Fig. 1 The role of CR in promoting the establishment of population inversion for low threshold lasing. a TEM images of a series of monodispersed $24 \mathrm{~nm}$ UCNPs. Scale bar $100 \mathrm{~nm}$. b-d Schematic illustration of the simplified energy level diagram and the role of doping concentration of $\mathrm{Tm}^{3+}$ in establishing the $\mathrm{CR}$ enabled population inversion of ${ }^{3} \mathrm{H}_{4}$ level, compared to the ground level.
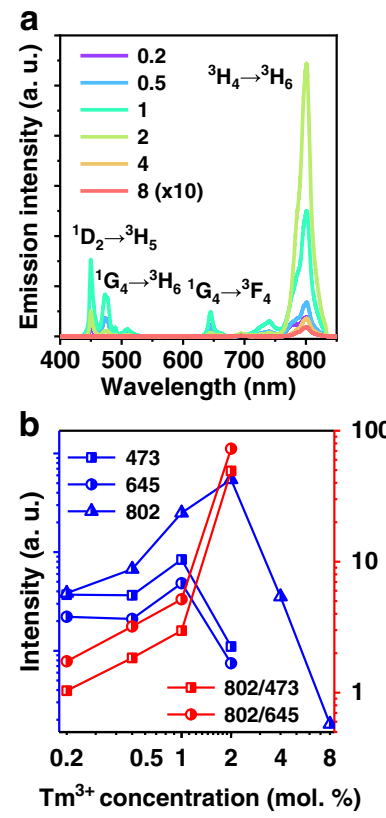

C

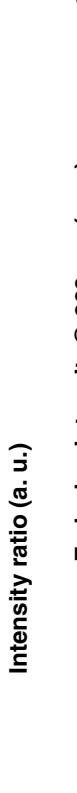

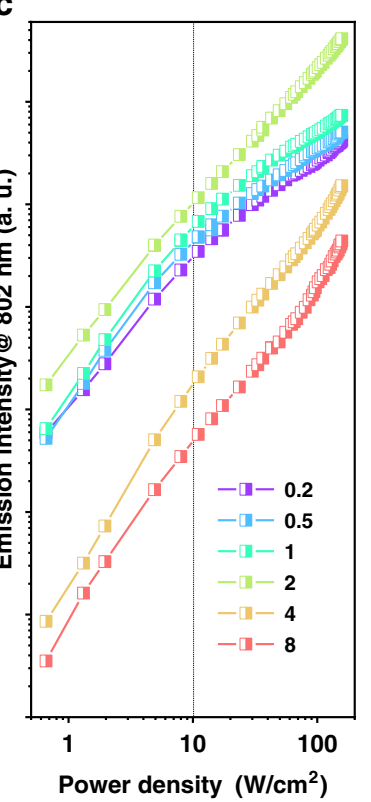

d

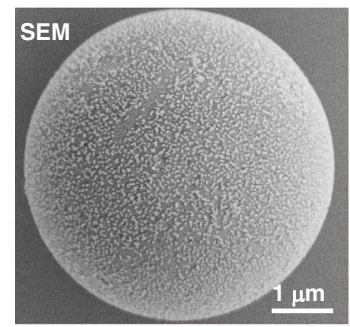

e
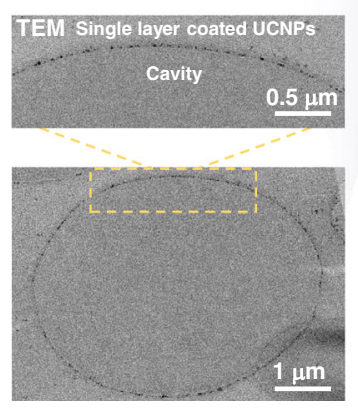

f

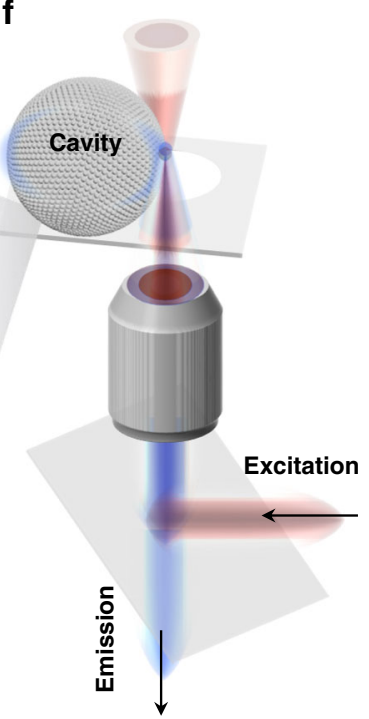

Fig. 2 Power dependent emission characteristics of upconversion nanoparticles with tunable doping concentrations and self-assembled UCNPs as the

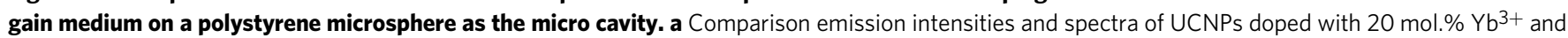

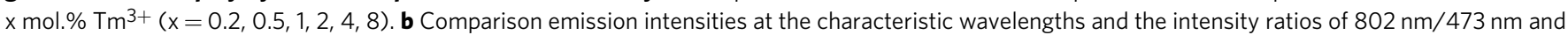
$802 \mathrm{~nm} / 645 \mathrm{~nm}$. Both results displayed in (a, b) were acquired at the excitation power density of $100 \mathrm{~W} / \mathrm{cm}^{2}$. c Power dependent emission intensity

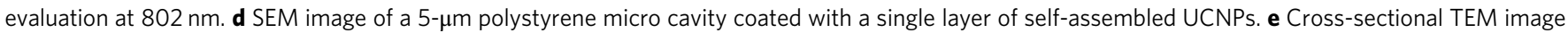
of micro cavity showing the distribution of UCNPs on the surface. $\mathbf{f}$ Excitation and detection scheme (see Supplementary Fig. 5 for the details of a purposebuilt imaging system).

and cross-sectional TEM measurements of a $5-\mu \mathrm{m}$ polystyrene microsphere, showing the single layer of $2 \mathrm{~mol} \% \mathrm{Tm}^{3+}$ doped UCNPs on the surface of the cavity through the electrostatic force assembly (see zeta potential data in Supplementary Fig. 9). Microsphere confines the light through total internal reflection and supports both transverse electric (TE) and transverse magnetic (TM) propagating modes with high quality $(\mathrm{Q})$ factor, e.g., the $\mathrm{Q}$ factor for a $750 \mu \mathrm{m}$ fused-silica microsphere could even reach $\sim 10^{9} 29-32$. When nanoparticles are coated onto the surface, the increased roughness induces scattering losses and reduces Q 
a
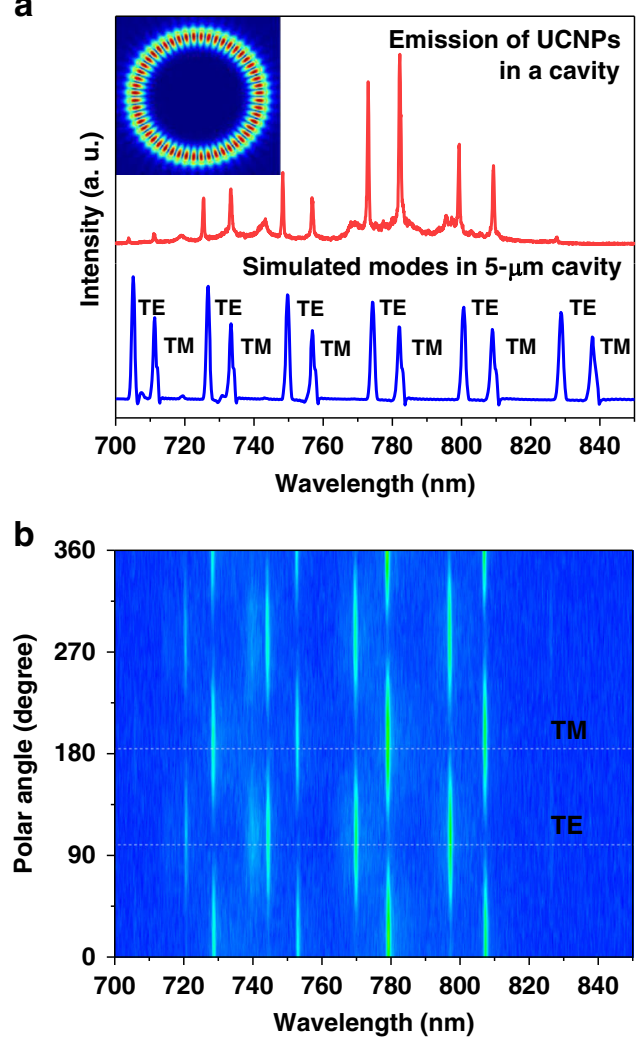

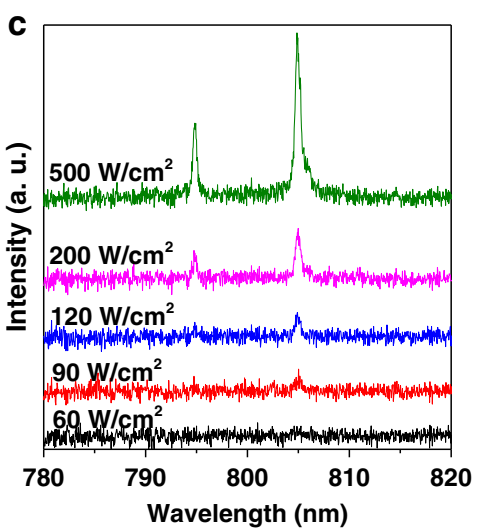

d

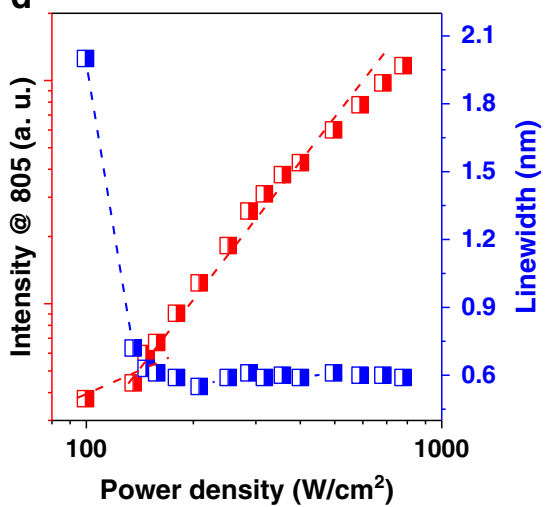

e

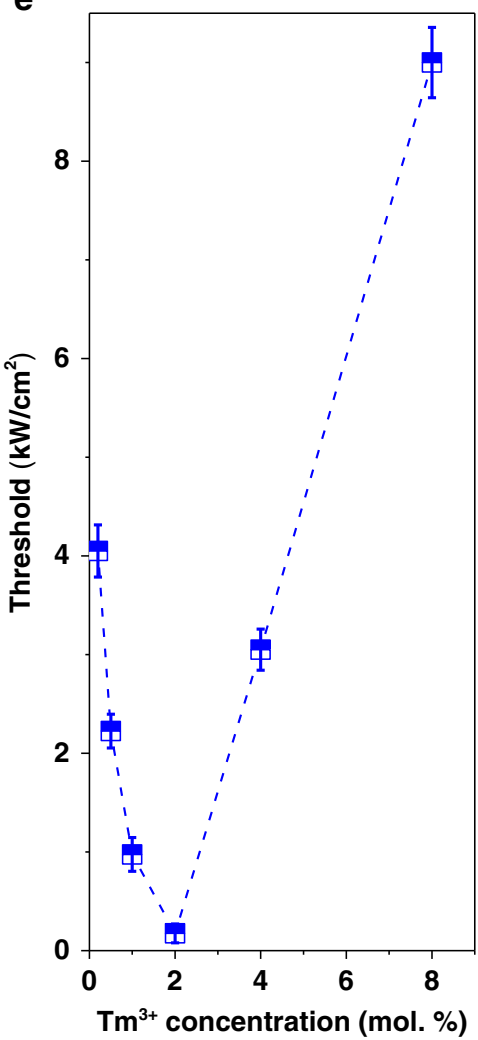

Fig. 3 Characteristics of low threshold upconversion lasing emissions from a single layer of self-assembled UCNPs in a polystyrene microsphere. a Numerical simulations of resonance spectrum and experimental emission spectrum. The inset shows a numerical simulation of the electrical-field distributions at $800 \mathrm{~nm}$ within a major plane. $\mathbf{b}$ Emission polarization angle-dependent intensities of the lasing peaks. $\mathbf{c}$ Power dependent upconversion emission spectra showing the gradual appearance of lasing peaks. $\mathbf{d}$ The pumping power-dependent plots of emission intensities and spectral linewidth narrowing, showing the onset of upconversion lasing emissions. e $\mathrm{Tm}^{3+}$ concentration-dependent thresholds for the onsets of upconversion lasing emissions.

factors $^{33}$, therefore homogeneous coatings avoid aggregations and minimize scattering.

Figure $3 \mathrm{a}$ shows the multi-mode lasing emissions under the pumping power of $10 \mathrm{~kW} / \mathrm{cm}^{2}$ and simulated optical modes of the cavity at $800 \mathrm{~nm}$ (see the details of Finite difference time domain simulations in Supplementary information and Supplementary Figs. 10 and 11 ). The $5-\mu \mathrm{m}$ microsphere with a refractive index of 1.59 leads to an effective coupling of upconversion emissions within the cavity modes. The full width at halfmaximum (FWHM) of the sharp lasing peaks is calculated to be $0.54 \mathrm{~nm}$ using the Lorentz function fitting. According to $\mathrm{Q} \approx \lambda_{0} /$ $\Delta \lambda$, where $\lambda_{0}$ and $\Delta \lambda$ are the centre wavelength and FWHM of the peak profile, the quality factor $Q$ is estimated as $\sim 1500$. Both the narrow FWHM and high Q benefit from the small size of ca. 24 $\mathrm{nm}$ of the UCNPs and the homogenous coating of a single layer of UCNPs (as the contrast, see the results of uneven coating in Supplementary Fig. 12). The inset in Fig. 3a and Supplementary Fig. 10 indicate that the jacinth-shaded region with high field intensity overlaps the coating layer of UCNPs.

The characterized polarization properties of both the TE and TM mode emissions exhibiting linear polarization with orthogonal periodicity as observed by the different polarization angles collection in Fig. 3b. The free spectral range (FSR) between two adjacent TE modes or TM modes fit well with the theoretical value $\left(\Delta \lambda_{\mathrm{FSR}}=\frac{\lambda^{2}}{2 \pi \mathrm{R}}, \mathrm{R}\right.$ is the radius of microcavity). Noting that the slight difference of experimental lasing peaks is attributed to the imperceptible slight variations of each cavity, as the mode position will move more than $8 \mathrm{~nm}$ when the size of this cavity changes for only $1 \%$ (Supplementary Fig. 11).
The transition from below, near, and at the threshold lasing emissions are seen in Fig. $3 c$ when increasing the pumping powers to the onset of lasing emissions with the characteristic sharp and regularly spaced emission peaks. The narrow peaks that indicative of increased coherence and laser emissions are emerging above the threshold at pump intensities of $120 \mathrm{~W} / \mathrm{cm}^{2}$ for the laser mode at $805 \mathrm{~nm}$. Both the slopes for the intensity and linewidth curves (Fig. 3d) display a non-linear change characteristic at the ultra-low pumping threshold of $\sim 150 \mathrm{~W} / \mathrm{cm}^{2}$ achieved for the homogeneously coated $2 \mathrm{~mol} . \% \mathrm{Tm}^{3+}$ doped UCNPs sample. Figure $3 \mathrm{e}$ shows $2 \mathrm{~mol} \% \mathrm{Tm}^{3+}$ doped UCNPs as the most effective gain medium as a result of the CR induced efficient population inversion at low pumping power (for the comparison characterizations of other UCNPs samples see Supplementary Fig. 13). Note that the accurate laser threshold should be ideally determined by using the absorbed pump power instead of incident pump power. We reason that the actual laser thresholds should be much lower than the current values once the absorbed power could be measured, though it's inaccessible in our experimental conditions.

Although the larger nanoparticles often produce stronger brightness, the size-induced scattering losses cannot be neglected. As shown in Fig. $4 \mathrm{a}-\mathrm{d}$, with the increase of nanoparticle size, from $24 \mathrm{~nm}$, to $43 \mathrm{~nm}$ and $51 \mathrm{~nm}$ the FWHM in the lasing spectra increases from $\sim 0.5 \mathrm{~nm}$ to $0.8 \mathrm{~nm}$ and $\sim 1.2 \mathrm{~nm}$, with the increased spontaneous emission background (Fig. 4e). Though larger sized UCNPs are brighter due to the reduced degree of surface-quenching, by testing more than 20 micro cavity lasers, the Q factor reduces from $\sim 1900$ to $\sim 700$ with the increased size of UCNPs (Fig. 4f). The reduction in the Q factor indicates the 

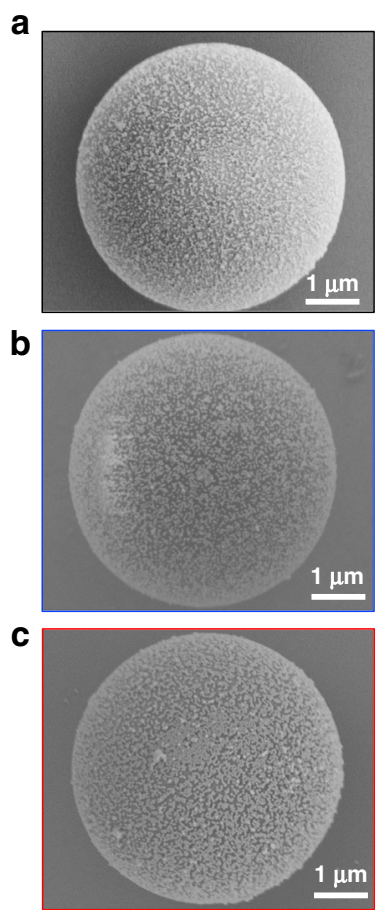

d 20

25
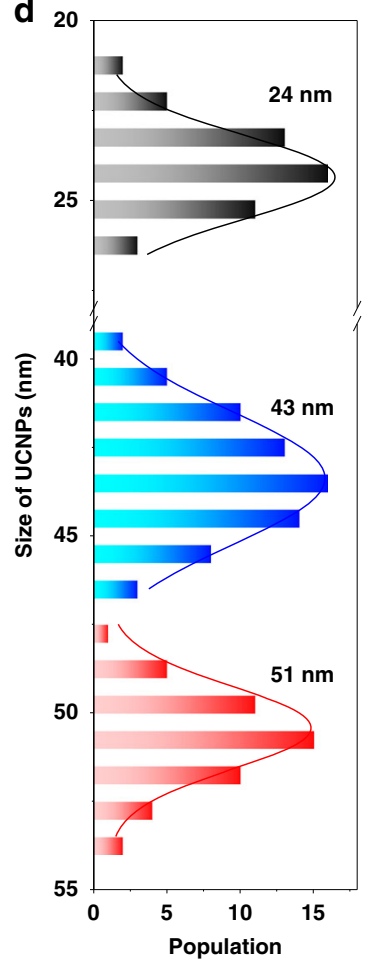

e

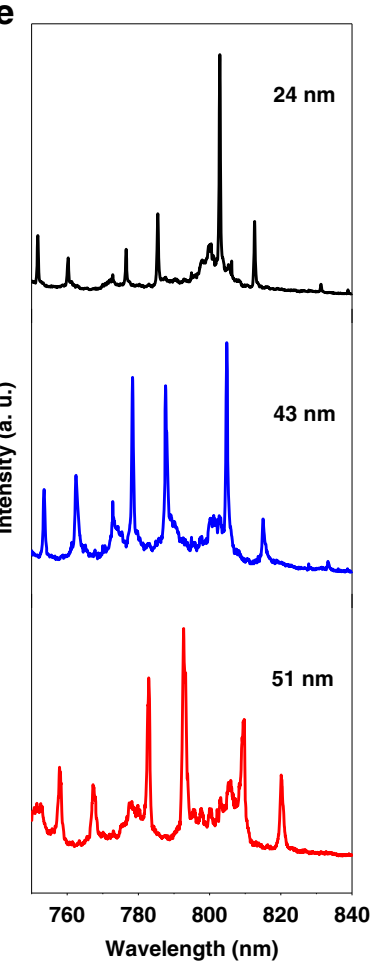

f

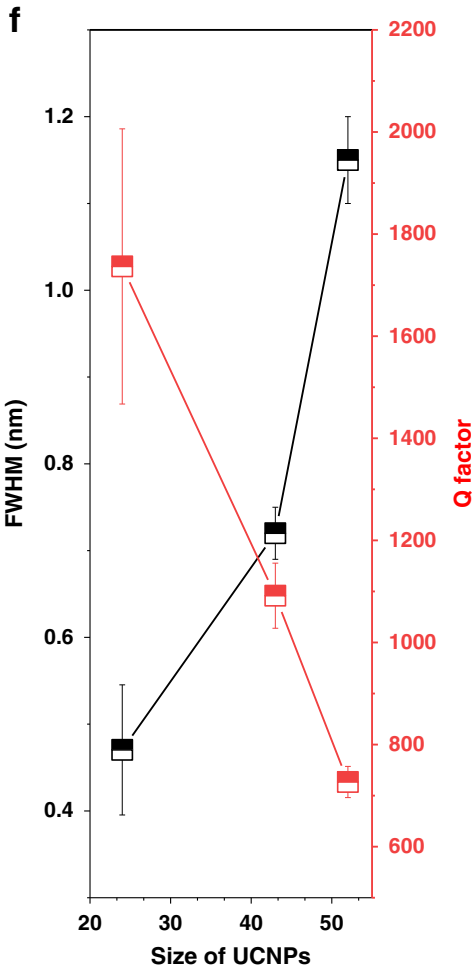

Fig. 4 Size-dependent quality $(Q)$ factors caused by the scattering losses when coating a single layer of nanoparticles in the micro cavity. a-c $S E M$ images of microspheres coated with $\mathrm{NaYF}_{4}: 20 \% \mathrm{Yb}^{3+}, 2 \% \mathrm{Tm}^{3+}$ nanoparticles with sizes of $24 \mathrm{~nm}, 43 \mathrm{~nm}$, and $51 \mathrm{~nm}$. d Size distributions of UCNPs used in (a-c). Related e emission spectra f FWHM and Q factor of UCNPs coated micro cavities in (a-c).

increased size-induced scattering loss. When the size of UCNPs becomes too large, the catchment area by the evanescent field of micro cavity modes also reduces (Supplementary Figs. 10 and 14).

Characterization of single nanocrystal lasing. Figure $5 a, b$ further show that lanthanide ions doped single UCNPs are optically uniform and sufficiently bright, as there are averaged $23640 \pm 832$ photon counts per 50 milliseconds from single nanoparticles under a confocal microscoy, which are suitable for single particle lasing in Fig. 5c. We demonstrate that CR assisted population inversion in single UCNPs as a highly efficient gain medium, which can be widely used in any photonics cavities for single nanoparticle lasers. Single $43 \mathrm{~nm} \mathrm{NaYF}_{4}$ : 20 mol.\% $\mathrm{Yb}^{3+}-2 \mathrm{~mol} . \%$ $\mathrm{Tm}^{3+}$ UCNPs can lase at $808 \mathrm{~nm}$ with the sharp peaks and narrow FWHM of $\sim 0.45 \mathrm{~nm}$ once in a cavity (Fig. $5 \mathrm{c}-\mathrm{f}$ ), which preserves the Q-factors of the cavity since scattering losses are reduced to the minimum. Due to the reduced scattering from single UCNP, it allows to use of bigger particles and increase the total gain, compared to the uniform $24 \mathrm{~nm}$ single layer coating.

Conclusion. We have achieved ultra-low threshold lasing by controlling $\mathrm{CR}$ in an upconversion energy transfer system and thereby the easy establishment of population inversion in single UCNPs. By using the doping-concentration-optimized UCNPs as the gain medium and single layer coating of monodispersed UCNPs on the cavity with minimized scattering loss, strong absorption and high efficiencies for both upconversion and population inversion establishment have been achieved. Employing a 5- $\mu \mathrm{m}$ cavity and homogenous coating architecture, we have achieved lasing emissions with a threshold of $\sim 150 \mathrm{~W} / \mathrm{cm}^{2}$, nearly two orders of magnitude lower than the recently reported benchmark value of $14 \mathrm{~kW} / \mathrm{cm}^{2}$. We have further verified that the size of single nanoparticle does not affect the $\mathrm{Q}$ factor of micro cavity lasing, with an FWHM of $\sim 0.45 \mathrm{~nm}$ achieved for upconversion lasing emissions from a single $43 \mathrm{~nm}$ nanoparticle. This study suggests great potential to using the concentration tunable UCNPs as an efficient gain medium for room temperature CW microscale and nanoscale lasers. The single upconversion nanocrystal lasing offers prospects to achieve NIR pumped antiStokes nanolaser platform for a variety of practical applications, such as intracellular tagging and imaging ${ }^{34}$. A further reduction of the threshold values and mode volumes is achievable by optimizing the combination strategy between the gain medium and cavity, and selecting higher-Q cavities, e.g., plasmonic nanocavity, Spaser, hyperbolic metacavity, photonic crystals or photonic topological insulator array cavities ${ }^{35,36}$. The CR mediated lanthanides energy transfer system might be embedded in semiconducting matrixes to achieve electrical pumped emission and further expand low threshold CW lasers via electrical pumping, which are more compatible with current standard technologies 37 .

\section{Methods}

Upconversion nanoparticles synthesis. The uniform oleic-acid-capped nanoparticles $\left(\beta-\mathrm{NaYF}_{4}: 20 \% \mathrm{Yb}, x \% \mathrm{Tm}^{3+}\right)$ were synthesized by the coprecipitation method. A typical procedure is as follows: $\mathrm{YCl}_{3} \cdot 6 \mathrm{H}_{2} \mathrm{O}(0.8-x \mathrm{mmol}), \mathrm{YbCl}_{3} \cdot 6 \mathrm{H}_{2} \mathrm{O}$ $(0.2 \mathrm{mmol})$ and $\mathrm{TmCl}_{3} \cdot 6 \mathrm{H}_{2} \mathrm{O}(x \mathrm{mmol})$ were added into a $50 \mathrm{~mL}$ three-necked flask containing $6 \mathrm{~mL}$ oleic acid $(\mathrm{OA})$ and $15 \mathrm{~mL}$ 1-octadecene (ODE). The mixture was first heated to $160^{\circ} \mathrm{C}$ under argon for $30 \mathrm{~min}$ to form a transparent solution and remove residual water. The solution was cooled down to room temperature, and $10 \mathrm{~mL}$ of a methanol solution containing $\mathrm{NaOH}(2.5 \mathrm{mmol})$ and $\mathrm{NH}_{4} \mathrm{~F}(4 \mathrm{mmol})$ was slowly dropped into the flask and stirred for $30 \mathrm{~min}$. Then, the solution was heated to $70-80^{\circ} \mathrm{C}$ and maintained for $30 \mathrm{~min}$ to evaporate methanol. Subsequently, the solution was heated to $300^{\circ} \mathrm{C}$ and maintained for $1 \mathrm{~h}$ under argon atmosphere. After cooling down to room temperature, the resulting products were precipitated by ethanol and collected by centrifugation at $6000 \mathrm{rpm}$ for $5 \mathrm{~min}$. The precipitate was then purified with ethanol three times, and finally dispersed in cyclohexane for further use.

Surface modification for water-soluble upconversion nanoparticles. In a typical procedure, the nanoparticles were first precipitated by adding ethanol $(2.0 \mathrm{~mL})$ to a cyclohexane colloidal solution of the OA coated hydrophobic $\beta$ - 
a

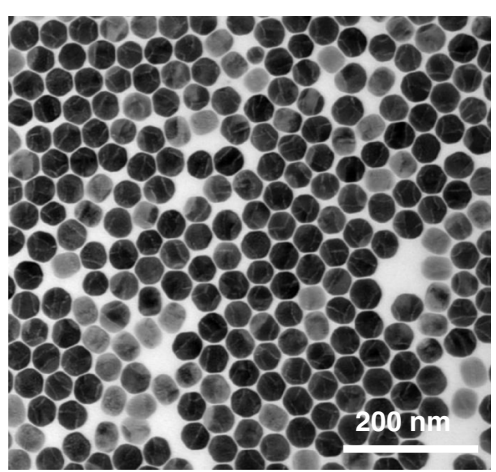

b
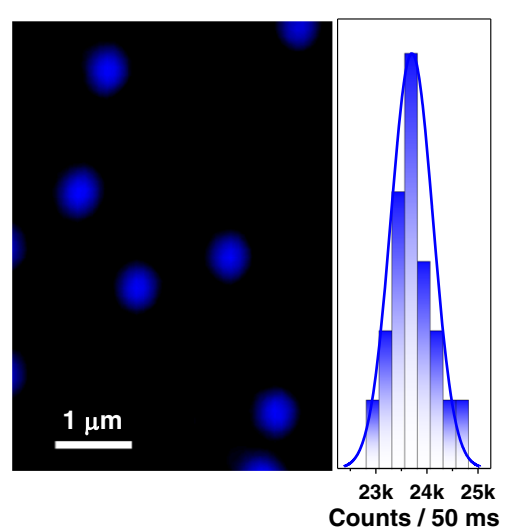

C

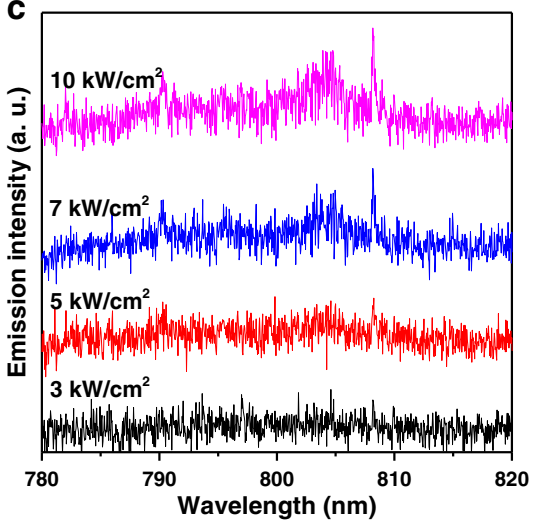

d

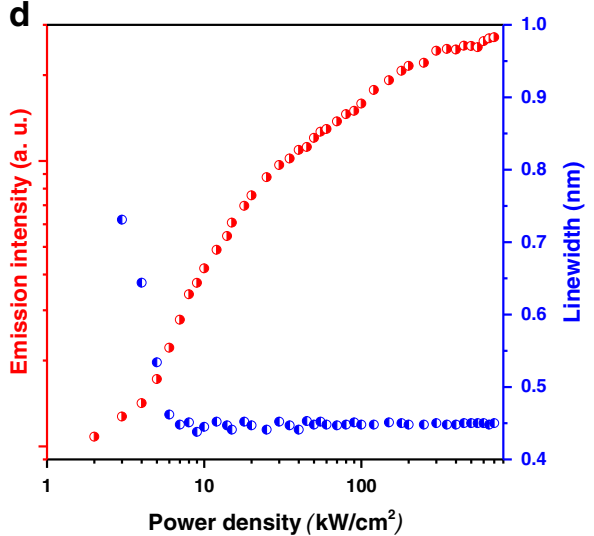

Power density $\left(\mathbf{k W} / \mathrm{cm}^{2}\right)$

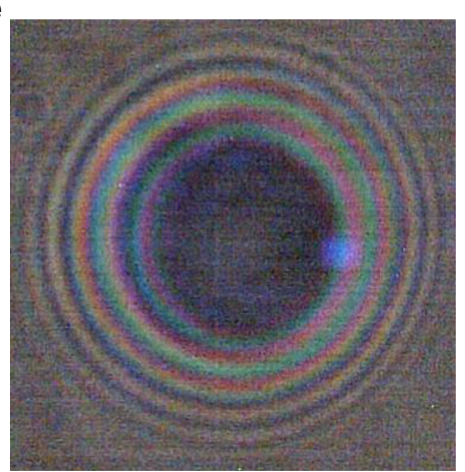

f

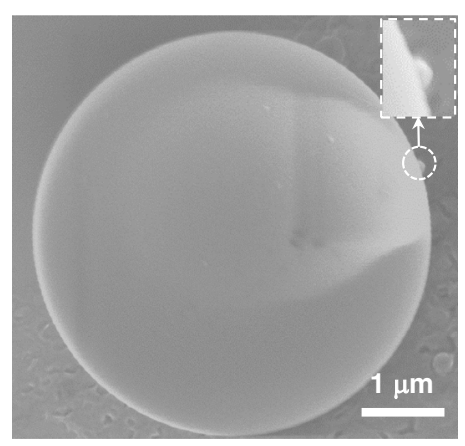

Fig. 5 Lasing emissions from a single nanocrystal. a TEM image of $43 \mathrm{~nm}$ monodispersed $\mathrm{NaYF}_{4}: 20 \% \mathrm{Yb}^{3+}, 2 \% \mathrm{Tm}^{3+}$ nanocrystals. b Point-scanning confocal microscopic image of single UCNPs (in pseudocolour) and their brightness distribution at an excitation power density of $100 \mathrm{~kW} / \mathrm{cm}^{2}$. $\mathbf{c}$ Power dependent upconversion emission spectra showing the gradual appearance of lasing emission peaks at $808 \mathrm{~nm}$. $\mathbf{d}$ The pumping power-dependent plots of emission intensities and spectral linewidth narrowing (peak@808 nm), showing the onset of single UCNP's lasing emissions. e, $\mathbf{f}$ Wide field image (d) and SEM image (e) of a single UCNP in a micro cavity.

$\mathrm{NaYF}_{4}: 20 \% \mathrm{Yb}, x \% \mathrm{Tm}^{3+}$ nanoparticles $(1 \mathrm{~mL}, 10 \mathrm{mg} / \mathrm{mL})$ and then collected by centrifugation at $6000 \mathrm{rpm}$ for $5 \mathrm{~min}$. The obtained nanoparticles were re-dispersed in a mixed solution of ethanol $(1 \mathrm{~mL})$ and $\mathrm{HCl}(1 \mathrm{~mL}, 2 \mathrm{M})$ upon sonication for 5 $\mathrm{min}$. The ligand free nanoparticles were collected by centrifugation at $14680 \mathrm{rpm}$ for $10 \mathrm{~min}$ and re-dispersed in deionized water $(1 \mathrm{~mL})$.

Fabrication of upconverting microlasers. Upconverting microlasers were produced through a solution process. In a typical procedure, $10 \mu \mathrm{L}$ PS microbeads ( $10 \%$ solids) were first dispersed in $1000 \mu \mathrm{L}$ DI water upon sonication for $5 \mathrm{~min}$, then $7 \mu \mathrm{L}$ ligand free water-soluble UCNPs $(8 \mathrm{mg} / \mathrm{mL})$ were mixed together with PS microbeads upon sonication. Then, this mixed solution was kept for a gentle shake $(750 \mathrm{rpm})$ for $2 \mathrm{~h}$ before centrifugation. These UCNPs coated microbeads were further washed with ethanol and water for three times, and finally were redispersed in water. Noting that, the coated surface and thickness can be controlled precisely by tuning the concentration ratio of microbeads and nanoparticles.

\section{Characterization and simulation}

TEM. Transmission electron microscope (TEM) measurements were performed using a FEI Tecnai T20 instrument with an operating voltage of $120 \mathrm{kV}$. The samples for TEM analysis were prepared by placing a drop of a dilute suspension of nanoparticles onto carbon-coated copper grids. For the cross-sectional TEM images, we first embedded the single layer UCNPs coated microcavity in epoxy (353ND), and then cut the microspheres using Leica microtome to get ultrathin slices.

SEM. The morphology of microcavity coated with UCNPs was characterized via scanning electron microscope (SEM) imaging (Supra 55VP, Zeiss) operated at $3.00 \mathrm{kV}$.

Zeta potential. The measurement of zeta potential ( $\zeta$-potential) was carried out by Zetasizer (Malvern Panalytical).

Confocal microscopy and spectroscopy. The inverted confocal optical system was built on a sample scanning configuration employing a 3D piezo stage. A singlemode fiber-coupled $980 \mathrm{~nm}$ diode laser was used as the excitation source and was focused onto the sample through an oil-immersion objective lens (UPlanAPO, Olympus; $\times 100, \mathrm{NA}=1.4$ ). The emission from sample was collected by the same objective lens then refocused into an optical fiber that has a core size matching with system Airy disk. A single photon counting avalanche diode (SPAD) detector was connected to the collection optical fiber to detect the emission intensity. The spectra were measured with a fiber-coupled spectrometer (Andor) with a grating of 1200 grooves $/ \mathrm{mm}$ (resolution: $0.21 \mathrm{~nm}$, data collection step: $\sim 0.04 \mathrm{~nm}$ ). And the polarization performance of this upconverting microlaser was characterized by rotating a $\lambda / 2$ plate @808 nm while cooperated with a polarizer $(620-1000 \mathrm{~nm})$

Finite difference time domain simulations. Numerical simulations of our microcavity were performed using Lumerical FDTD solutions. For the simulation of the electrical-field distributions and resonance spectrum, the perfectly matched layer (PML) was set as the boundary condition with a simulation region of $6 \mu \mathrm{m} \times$ $6 \mu \mathrm{m} \times 6 \mu \mathrm{m}$; the monitored wavelength was $400-1000 \mathrm{~nm}$; the meshes order was $10 \mathrm{~nm}$; the light type was a dipole source. The simulated resonance spectrum and electric field distribution of microcavity were calculated separately, as shown in Fig. 3a. The cavity modes in Supplementary Fig. 11 were achieved while changing the sizes of the microcavity. The electrical-field distributions at $\sim 800 \mathrm{~nm}$ mode was plotted along the diameter.

\section{Data availability}

All the relevant data are available from the corresponding authors upon reasonable request.

Received: 27 February 2020; Accepted: 21 October 2020; Published online: 01 December 2020

\section{References}

1. Auzel, F. Upconversion and anti-stokes processes with $\mathrm{f}$ and $\mathrm{d}$ ions in solids. Chem. Rev. 104, 139-173 (2004). 
2. Liu, Y. et al. Amplified stimulated emission in upconversion nanoparticles for super-resolution nanoscopy. Nature 543, 229-233 (2017).

3. Zhao, J. et al. Single-nanocrystal sensitivity achieved by enhanced upconversion luminescence. Nat. Nanotechnol. 8, 729 (2013).

4. Jackson, S. D. Cross relaxation and energy transfer upconversion processes relevant to the functioning of $2 \mu \mathrm{m} \mathrm{Tm}{ }^{3+}$-doped silica fibre lasers. Opt. Commun. 230, 197-203 (2004)

5. Hebert, T., Wannemacher, R., Macfarlane, R. \& Lenth, W. Blue continuously pumped upconversion lasing in Tm: $\mathrm{YLiF}_{4}$. Appl. Phys. Lett. 60, 2592-2594 (1992).

6. Chen, X., Sun, T. \& Wang, F. Lanthanide-based luminescent materials for waveguide and lasing. Chem.- Asian J. 15, 21-33 (2020).

7. Ermeneux, F. et al. Growth and fluorescence properties of $\mathrm{Tm}^{3+}$ doped $\mathrm{YVO}_{4}$ and $\mathrm{Y}_{2} \mathrm{O}_{3}$ single crystals. Optical Mater. 8, 83-90 (1997).

8. Zhang, L. et al. Crystal growth, optical spectroscopy and laser action of $\mathrm{Tm}^{3+}$-doped monoclinic magnesium tungstate. Opt. Express 25, 3682-3693 (2017).

9. Wen, S. et al. Advances in highly doped upconversion nanoparticles. Nat. Commun. 9, 2415 (2018)

10. Lu, Y. Q. et al. Tunable lifetime multiplexing using luminescent nanocrystals. Nat. Photonics 8, 33-37 (2014).

11. Wang, F. et al. Simultaneous phase and size control of upconversion nanocrystals through lanthanide doping. Nature 463, 1061 (2010).

12. Wang, F. et al. Tuning upconversion through energy migration in core-shell nanoparticles. Nat. Mater. 10, 968 (2011).

13. Liu, D. et al. Three-dimensional controlled growth of monodisperse sub-50 $\mathrm{nm}$ heterogeneous nanocrystals. Nat. Commun. 7, 10254 (2016).

14. Gargas, D. J. et al. Engineering bright sub-10-nm upconverting nanocrystals for single-molecule imaging. Nat. Nanotechnol. 9, 300-305 (2014).

15. Liu, Q. et al. Single upconversion nanoparticle imaging at sub-10 $\mathrm{W} \mathrm{cm}-2$ irradiance. Nat. Photonics 12, 548-553 (2018).

16. Tian, B. et al. Low irradiance multiphoton imaging with alloyed lanthanide nanocrystals. Nat. Commun. 9, 3082 (2018).

17. Gu, Y. et al. High-sensitivity imaging of time-domain near-infrared light transducer. Nat. Photonics 13, 525-531 (2019).

18. Zhan, Q. et al. Achieving high-efficiency emission depletion nanoscopy by employing cross relaxation in upconversion nanoparticles. Nat. Commun. 8 , 1058 (2017).

19. Wei, W. et al. Cross relaxation induced pure red upconversion in activatorand sensitizer-rich lanthanide nanoparticles. Chem. Mater. 26, 5183-5186 (2014).

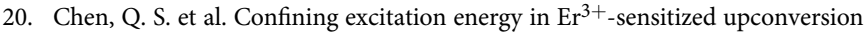
nanocrystals through $\mathrm{Tm}^{3+}$-mediated transient energy trapping. Angew. Chem. Int. Ed. 56, 7605-7609 (2017).

21. Zhong, Y. et al. Boosting the down-shifting luminescence of rare-earth nanocrystals for biological imaging beyond $1500 \mathrm{~nm}$. Nat. Commun. 8, 737 (2017)

22. Wang, J., Wang, F., Wang, C., Liu, Z. \& Liu, X. Single-band upconversion emission in lanthanide-doped $\mathrm{KMnF}_{3}$ nanocrystals. Angew. Chem. Int. Ed. 50, 10369-10372 (2011)

23. Fernandez-Bravo, A. et al. Continuous-wave upconverting nanoparticle microlasers. Nat. Nanotechnol. 13, 572 (2018).

24. Hulst, H. C. \& van de Hulst, H. C. Light scattering by small particles (Courier Corporation, 1981)

25. Bohren, C. F. \& Huffman, D. R. Absorption and scattering of light by small particles (1983)

26. Misiak, M., Prorok, K., Cichy, B., Bednarkiewicz, A. \& Stręk, W. Thulium concentration quenching in the up-converting $\alpha-\mathrm{Tm}^{3+} / \mathrm{Yb}^{3+} \mathrm{NaYF}_{4}$ colloidal nanocrystals. Optical Mater. 35, 1124-1128 (2013).

27. Yu, D. et al. Understanding and tuning blue-to-near-infrared photon cutting by the $\mathrm{Tm}^{3+} / \mathrm{Yb}^{3+}$ couple. Light.: Sci. Appl. 9, 107 (2020).

28. Chen, C. et al. Multi-photon near-infrared emission saturation nanoscopy using upconversion nanoparticles. Nat. Commun. 9, 3290 (2018).

29. Vahala, K. J. Optical microcavities. Nature 424, 839 (2003).

30. He, L., Özdemir, Ş. K. \& Yang, L. Whispering gallery microcavity lasers. Laser Photonics Rev. 7, 60-82 (2013).
31. Jiang, X. F., Qavi, A. J., Huang, S. H. \& Yang, L. Whispering-gallery sensors. Matter 3, 371-392 (2020).

32. Gorodetsky, M. L., Savchenkov, A. A. \& Ilchenko, V. S. Ultimate Q of optical microsphere resonators. Opt. Lett. 21, 453-455 (1996).

33. Boriskina, S. V., Benson, T. M., Sewell, P. \& Nosich, A. I. Spectral shift and Q change of circular and square-shaped optical microcavity modes due to periodic sidewall surface roughness. J. Optical Soc. Am. B 21, 1792-1796 (2004).

34. Martino, N. et al. Wavelength-encoded laser particles for massively multiplexed cell tagging. Nat. Photonics 13, 720-727 (2019).

35. Jeong, K. Y. et al. Recent progress in nanolaser technology. Adv Mater, 202001996 (2020).

36. Garcia-Vidal, F. J. \& Moreno, E. Lasers go nano. Nature 461, 604-605 (2009).

37. Ma, R.-M. \& Oulton, R. F. Applications of nanolasers. Nat. Nanotechnol. 14, 12-22 (2019).

\section{Acknowledgements}

This project is primarily supported by ARC Discovery Early Career Researcher Award Scheme (J.Z., DE180100669), China Scholarship Council Scholarships (Y.S.: No. 201706120322), National Natural Science Foundation of China (NSFC, 61729501), Major International (Regional) Joint Research Project of NSFC (51720105015), Science and Technology Innovation Commission of Shenzhen (KQTD20170810110913065), and Australia China Science and Research Fund Joint Research Centre for Point-of-Care Testing (ACSRF658277, SQ2017YFGH001190).

\section{Author contributions}

Y.S., J.Z., and D.J. conceived the project and designed experiments. J.Z., C.Y., L.J., and D.J. supervised the project. Y.S. and Y.C. synthesized the upconversion nanoparticles. Y.S., F.W. and J.Z. conducted the optical setup. Y.S. fabricated the microlasers and performed the characterizations. Y.S., J.Z., A.F., and D.J. analyzed the results, prepared the figures and wrote the paper. All authors contributed to discussion, interpreting the data and the writing.

\section{Competing interests}

The authors declare no competing interests.

\section{Additional information}

Supplementary information is available for this paper at https://doi.org/10.1038/s41467 020-19797-4.

Correspondence and requests for materials should be addressed to J.Z.

Peer review information Nature Communications thanks the anonymous reviewers for their contribution to the peer review of this work. Peer reviewer reports are available.

Reprints and permission information is available at http://www.nature.com/reprints

Publisher's note Springer Nature remains neutral with regard to jurisdictional claims in published maps and institutional affiliations.

Open Access This article is licensed under a Creative Commons Attribution 4.0 International License, which permits use, sharing, adaptation, distribution and reproduction in any medium or format, as long as you give appropriate credit to the original author(s) and the source, provide a link to the Creative Commons license, and indicate if changes were made. The images or other third party material in this article are included in the article's Creative Commons license, unless indicated otherwise in a credit line to the material. If material is not included in the article's Creative Commons license and your intended use is not permitted by statutory regulation or exceeds the permitted use, you will need to obtain permission directly from the copyright holder. To view a copy of this license, visit http://creativecommons.org/licenses/by/4.0/. 